\title{
Taxonomic Study of the Subfamily Lycorininae (Hymenoptera: Ichneumonidae) from Korea
}

\author{
Jin-Kyung Choi ${ }^{1}$, Jin-Yeol Cha ${ }^{2}$, Jong-Wook Lee ${ }^{1, *}$ \\ 'Department of Life Sciences, Yeungnam University, Gyeongsan 712-749, Korea \\ ${ }^{2}$ Korea National Park Service, Seoul 121-717, Korea
}

\begin{abstract}
Korean species of the subfamily Lycorininae Cushman \& Rohwer are reviewed. This subfamily along with three species, Lycorina triangulifera Holmgren, 1859, L. spilonotae Chao, 1980, and L. ruficornis Kasparyan, 2007, are reported for the first time from Korea. In this genus, five species have been reported from the Eastern Palaearctic region, six species from China and two species from Japan. Some species of this subfamily is known as parasitoids of Crambidae and Tortricidae. We report three newly recorded species from Korea, Lycorina triangulifera, L. spilonotae, and L. ruficornis, and provide diagnoses with photographs of lycorinine species and a key to the Korean species.
\end{abstract}

Keywords: Korea, Lycorina triangulifera, Lycorina spilonotae, Lycorina ruficornis, taxonomy

\section{INTRODUCTION}

The subfamily Lycorininae Cushman \& Rohwer, 1920 is a small group with a single genus, Lycorina. It is a cosmopolitan genus with 30 described species (Yu et al., 2012). In this genus, five species have been reported from the Eastern Palaearctic region, six species from China, two species from Japan. This subfamily is easily distinguished from other ichneumonid families based on tergites with a triangular area by deep impressions (Fig. 1G, I); pronotum with anterior margin reflexed; and hypopygium of female large triangular in lateral view (Fig. 2C). The focal host range appears to be weakly concealed larva of micro-lepidopteran species. Some species have been reared from Crambidae in leaf-rolls or webs (Doerksen and Neunzig, 1974; Finlayson, 1976) and Tortricidae (Chao, 1980; Shaw, 2004).

In this study, we add three newly recorded species, Lycorina triangulifera Holmgren, L. spilonotae Chao, and L. ruficornis Kasparyan, to Korean ichneumonoids fauna and provide descriptions, a key to the Korean species, and photographs.

Materials used in this work were collected by sweeping and Malaise trap, after which they were deposited in the animal systematic laboratory of the Yeungnam University (YNU, Gyeongsan, Korea). Specimens were examined by a AxioCam

(c) This is an Open Access article distributed under the terms of the Creative Commons Attribution Non-Commercial License (http://creativecommons.org/ licenses/by-nc/3.0/) which permits unrestricted non-commercial use, distribution, and reproduction in any medium, provided the original work is properly cited.

pISSN 2234-6953 eISSN 2234-8190
MRc5 camera attached to a stereo microscope (Zeiss SteREO Discovery. V20; Carl Zeiss, Göttingen, Germany), processed using AxioVision SE64 software (Carl Zeiss), and optimized with a Delta imaging system (i-solution; IMT i-Solution Inc., Vancouver, Canada).

Abbreviations are as follows: TD, type depository; TS, type species; ZI, Zoological Institute, Academy of Sciences, Russia; NR, Naturhistoriska Riksmuseet, Sektionen für Entomologi, Stockholm, Sweden; UZM, Universitets Zoologiske Museum, Copenhagen, Denmark; USN, Dipartimento di Zoologia, Università degli Studi di Napoli, Via Mezzocannone 8, Napoli, Italy; FAFU, Fujian Agriculture \& Forestry University, Institute of Beneficial Insects, Fuzhou, Fujian, China; GW, Gangwon-do; GG, Gyeonggi-do; GN, Gyeongsangnam-do; JJ, Jeju-do.

\section{SYSTEMATIC ACCOUNTS}

Order Hymenoptera

Family Ichneumonidae Latreille, 1802

Subfamily Lycorininae Cushman \& Rohwer, 1920

Genus Lycorina Holmgren, 1859

Lycorina Holmgren, 1859: 126. TS: Lycorina triangulifera

*To whom correspondence should be addressed

Tel: 82-53-810-2376, Fax: 82-53-811-2376

E-mail: jwlee1@ynu.ac.kr 
Holmgren.

Amyx Schiødte, 1839: 309. TS: Amyx flavilabris Schiødte.

Toxophoroides Cresson, 1874: 406. TS: Lycorina? Apicalis Cresson.

Chlorolycorina Cushman, 1920: 7. TS: Glypta scitula Cushman.

Gonioglyphus Seyrig, 1932: 183. TS: Lycorina fici Seyrig.

\section{Key to the species of the subfamily Lycorininae from Korea}

1. Face almost yellow (Fig. 1E); posterior part of propodeum yellow; fore and mid coxa yellow, base of coxa slight brown; nervellus of hind wing intercepted on a little lower third of it (Fig. 2D); metasomal segments with yellow line to apex $($ Fig. $1 \mathrm{H}) \quad \ldots \ldots \ldots \ldots \ldots \ldots \ldots$ Lycorina spilonotae $\mathrm{C}$.

- Face almost black or with small yellow spots (Fig. 1D, F); propodeum black; fore and mid coxa black; nervellus of hind wing intercepted on lower fourth of it (Fig. 2E); metasomal segments black (Fig. 1G, I) …................. 2

2. Margin of clypeus almost round or weakly concave (Fig. 1D, E); fore femur straight (Fig. 2A); hind femur reddish Lycorina triangulifera $\mathrm{H}$.

- Margin of clypeus strongly emergence (Fig. 1F); fore femur very strongly curved (Fig. 2B); hind femur yellow

Lycorina ruficornis $\mathrm{K}$.

${ }^{1 *}$ Lycorina triangulifera Holmgren, 1859

Lycorina triangulifera Holmgren, 1859: 126. Type: female, TD: NR.

Amyx flavilabris (Schiødte, 1839): 309. Type: female, TD: UZM.

Glypta lycorinoides (Costa, 1886a): 40. Type: unknown, TD: USN.

Glypta sardoa (Costa, 1886b): 51. Type: unknown, TD: USN.

Material examined. Korea: 1 female, GG, Namyangju-si, Joan-myeon, Songchon-ri, Ungilsan, $37^{\circ} 34^{\prime} 43.2^{\prime \prime} \mathrm{N}, 127^{\circ} 18^{\prime}$ 37.5”E, 26 Jun-16 Jul 2009, Lim JO; 2 females, GG, Anyangsi, Manan-gu, Kwanag Arb., alt. 219 m, 37 $18^{\prime} 5.3^{\prime \prime} \mathrm{N}, 127^{\circ}$ 19'1.5”E, 5-19 Jul 2007, Lim JO; 1 male, GN, Milyang-si, Sajapyeong, 22 Jun 1993, Lee JW; 1 female, Daegu-si, Dalsegu, Daegok-dong, Daegu Arb., alt. $88 \mathrm{~m}, 35^{\circ} 47^{\prime} 48.6^{\prime \prime} \mathrm{N}, 128^{\circ}$ 31'33.5”E, 20 Jun-4 Jul 2012, Kang SG.

Diagnosis. Female. Fore wing 6.4-6.6 mm, body 7.0-7.2 $\mathrm{mm}$, ovipositor 3.0-3.2 mm long.

Color: Body black. Face with pairs yellow spots and behind margins of eyes with yellow line. Clypeus, palpi, central part of mandible, malar space, fore and mid legs except coxa, tegula, scutellum from behind and postscutellum yellow. Antennal flagellum ventrally and ovipositor brown. Hind trochantellus to femur reddish brown. Hind tibia except apically and ovipositor sheath except apically dark brown.

Head: Face moderately dense punctured, the distance between points longer than their diameter, 1.3 times as wide as high, central part of face convex laterally. Frons with longitudinal carina, concave above base of antennae, raised laterally. Clypeus moderately flat, with round apical edge, separated from face by transverse groove; clypeus and temple finely shagreened between points. Malar space 1.0 times as long as basal width of mandible. Flagellum with 27-29 segments, first segment 4.5 times as long as wide, apical segments square. Scape swollen, 1.7 times as wide as width of first flagellomere.

Mesosoma: Epomia present, strong, with upper end arcuate produced. Pronotum mediodorsally without deep transverse furrow that is crossed by a longitudinal 'bridge'. Middle lobe of mesoscutum in front vertically descending down. Pronotum loosely punctated. Notaulus shallow. Mesoscutum slightly longer than wide, 1.3 times as long as wide. Scutellum carinated and convex. Mesoscutum and scutellum sparsely punctured more than head. Subalar prominence convex, speculum separated and glabrous, with pit strong, epicnemial carina incomplete. Propodeum very short, anterior transverse carina present, propodeal spiracle small, oval, closer to lateral longitudinal carina than to pleural, basal area glabrous. Nervellus intercepted on lower fourth, discoidella vestigial. Hind wing with 10-11 distal hamuli. Leg slender, correlation between length of hind tarsal segments as $50: 16: 11: 7: 13$. Tarsal claws pectinate.

Metasoma: Depressed. Raised central triangular areas large and strongly transverse (Fig. 1G), terga quite closely and coarsely punctate. Ovipositor sheath 1.6 times as long as hind tibia.

Host. [Lepidoptera]: Gelechiidae, Anacampsis timidella (Capek, 1955), Tortricidae, Archips rosana (Sedivy, 1986; Jonaitis, 1992), Epiblema foenella (Uffeln, 1940), Epiblema sticticana (Aubert, 1978), Yponomeutidae, Yponomeuta malinella (Meyer, 1934), Yponometuta padella (Friese, 1963). [Coleoptera]: Cerambycidae, Saperda populnea (Kleine, 1909; Scheidter, 1917; Postner, 1954; Fulmek, 1968; UhthoffKaufmann, 1991).

Distribution. Korea (new record), Austria, Azerbaijan, Belarus, Belgium, Bulgaria, China, Czechoslovakia, Denmark, Estonia, Finland, France, Germany, Hungary, Ireland, Italy, Japan, Lithuania, Moldova, Mongolia, Netherlands, Norway, Poland, Romania, Russia, Slovakia, Sweden, Ukraine, United Kingdom. 

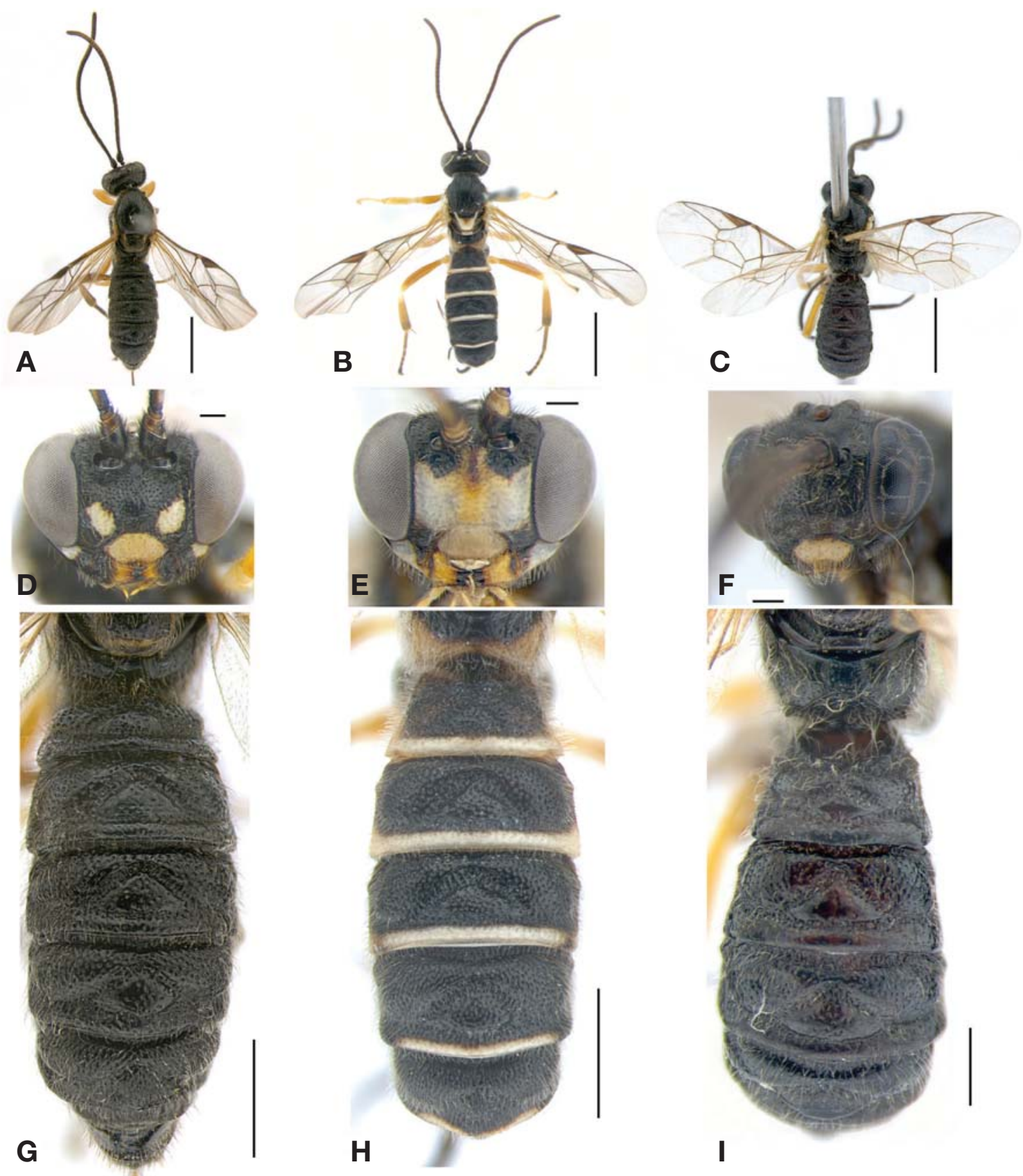

Fig. 1. Korean Lycorininae. A-C, Habitus in dorsal view; A, Lycorina triangulifera; B, L. spilonotae; C, L. ruficornis; $D-F$, Head in frontal view; D, L. triangulifera; E, L. spilonotae; F, L. ruficornis; G-I, Metasoma in dorsal view; G, L. triangulifera; H, L. spilonotae; $\mathrm{I}$, L. ruficornis. Scale bars: $\mathrm{A}-\mathrm{C}=2 \mathrm{~mm}, \mathrm{D}-\mathrm{F}=0.2 \mathrm{~mm}, \mathrm{G}, \mathrm{H}=1 \mathrm{~mm}, \mathrm{I}=0.5 \mathrm{~mm}$.

\section{${ }^{1 *}$ Lycorina spilonotae Chao, 1980}

Lycorina spilonotae Chao, 1980: 165. Type: female, TD: FAFU.

Material examined. Korea: 1 female, JJ, Jeju-si, Donggye- myeon, near Jeolmul, 25 Aug-2 Sep 2005, Sin CH.

Diagnosis. Female. Fore wing $5.8 \mathrm{~mm}$, body $6.9 \mathrm{~mm}$, ovipositor $2.8 \mathrm{~mm}$ long.

Color: Body black. Face, clypeus, malar space, fore and mid coxa to trochantellus, tegula, scutellum, postscutellum and

Korean name: ${ }^{1 *}$ 노란줄삼각배무늬맵시벌 (신칭) 

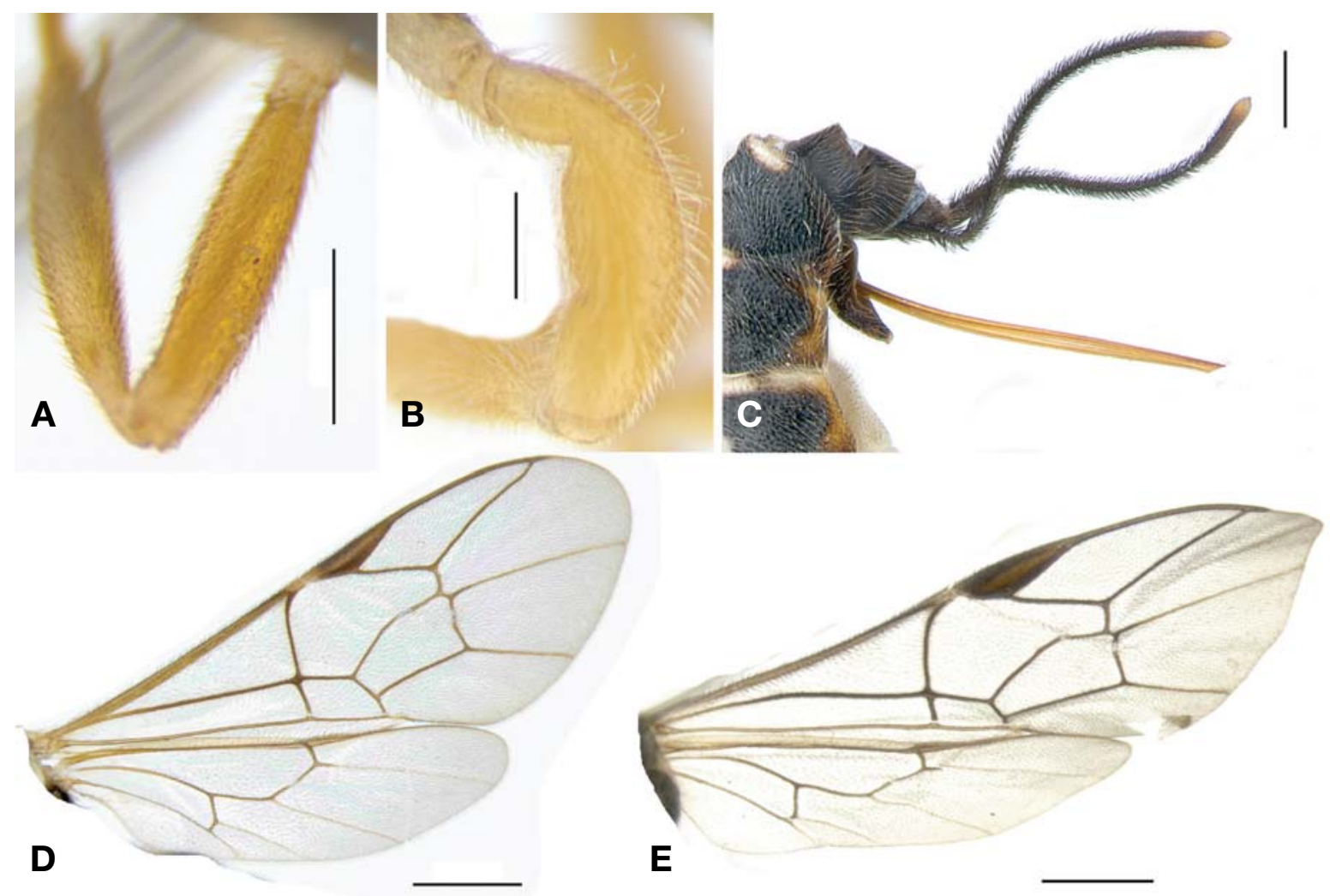

Fig. 2. $A, B$, Fore femur in lateral view; $A$, Lycorina triangulifera; $B, L$. ruficornis; $C$, Hypopygium and ovipositor sheath of $L$. spilonotae; $\mathrm{D}, \mathrm{E}$, Wings; $\mathrm{D}, \mathrm{L}$. spilonotae; $\mathrm{E}$, L. triangulifera. Scale bars: $\mathrm{A}, \mathrm{C}=0.5 \mathrm{~mm}, \mathrm{~B}=0.2 \mathrm{~mm}, \mathrm{D}, \mathrm{E}=1 \mathrm{~mm}$.

tergites apex lines apically yellow. Margin of eyes vertex in temple, mandible except apical teeth, palpi, fore and mid legs except base, half of pronotum yellow. Antennal flagellomeres dark brown but brown ventrally. Hind coxa dark brown. Hind leg brown except apex of tibia and tarsus dark brown. Ovipositor brown.

Head: Face and clypeus very sparsely punctuated, 1.1 times as wide as high, central part of face weakly convex laterally. Frons with longitudinal carina, concave above base of antennae, weakly raised laterally. Clypeus moderately flat, separated from face by transverse groove. Malar space 0.8 times as long as basal width of mandible. Ocelli area convex. Flagellum with 28 segments, first segment 3.4 times as long as wide, apical segments square. Scape swollen, 1.6 times as wide as width of first flagellomere.

Mesosoma: Epomia present, with upper end arcuate produced. Pronotum mediodorsally without deep transverse furrow that is crossed by a longitudinal 'bridge'. Pronotum with furrow and wrinkle, central part glabrous. Notaulus shallow. Mesoscutum slightly longer than wide, 1.3 times as long as wide. Scutellum carinated and convex. Mesoscutum closely punctated, scutellum sparsely punctured. Subalar prominence weakly concave, speculum convex and glabrous, with pit weak, epicnemial carina incomplete. Mesopleuron coarsely punctured except below of speculum finely shagreened. Propodeum very short, with scattered punctures, anterior transverse carina incomplete, posterior transverse carina distinct. Propodeal spiracle small, rounded, closer to pleural carina than to lateral longitudinal. Nervellus intercepted on a little lower third of it, discoidella vestigial. Hind wing with nine distal hamuli. Legs slender, correlation between length of hind tarsal segments as $36: 15: 10: 6: 11$. Tarsal claws pectinate.

Metasoma: Depressed. Raised central triangular areas large and strongly transverse (Fig. $1 \mathrm{H}$ ), terga very closely and coarsely punctate. Ovipositor sheath 1.1 times as long as hind tibia.

Host. [Lepidoptera]: Tortricidae, Adoxophyes orana (He et al., 1996), Spilonota lechriaspis (Chao, 1980; He et al., 1996).

Distribution. Korea (new record), China.

\section{$1 *$ Lycorina ruficornis Kasparyan, 2007}

Lycorina ruficornis Kasparyan, 2007: 429. Type: female, TD: ZI. 
Material examined. Korea: 1 female, GW, Yanggu-gun, Gachilbong, 31 May 1992, Lee JW.

Diagnosis. Female. Fore wing $5.5 \mathrm{~mm}$, body $6.5 \mathrm{~mm}$, ovipositor $2.8 \mathrm{~mm}$ long.

Color: Body black. Clypeus, palpi, mandible apically and tegula yellow. Fore and mid legs except coxa and trochanters, hind femur and tibia except apical yellow, coxa and trochanters black, hind tarsus dark brown. Ovipositor brown, ovipositor sheath except apically dark brown.

Head: Face sparsely punctuated, 1.2 times as wide as high, central part of face weakly convex laterally. Frons with longitudinal carina, concave above base of antennae, weakly raised laterally. Clypeus emarginated, weakly convex, separated from face by transverse carina. Malar space 0.9 times as long as basal width of mandible. Ocellar area convex. Flagellum with 26 segments, first segment 3.8 times as long as wide, apical segments square. Scape swollen, 1.8 times as wide as width of first flagellomere.

Mesosoma: Epomia present, with upper end arcuate produced. Pronotum mediodorsally without deep transverse furrow that is crossed by a longitudinal 'bridge'. Pronotum loosely punctated. Notaulus shallow. Mesoscutum slightly shorter than wide, 0.9 times as long as wide. Scutellum carinated. Mesoscutum closely punctated, scutellum with scattered punctures. Subalar prominence convex, speculum convex and glabrous, with strong pit, epicnemial carina incomplete. Mesopleuron sparsely punctured except below weakly rugosely punctured. Propodeum very short, with coarsely punctures, anterior transverse carina absent, posterior transverse carina distinct. Propodeal spiracle small, rounded, located middle of lateral area. Nervellus intercepted on a lower fourth, discoidella vestigial. Hind wing with nine distal hamuli. Leg slender but fore femur very strongly curved, correlation between length of hind tarsal segments as $10: 4: 3: 2: 4$. Tarsal claws pectinate.

Metasoma: Depressed. Raised central triangular areas large and strongly transverse (Fig. 1I), terga coarsely punctuate, first and second central triangular area very sparsely punctuated. Ovipositor sheath 1.3 times as long as hind tibia.

Host. Unknown.

Distribution. Korea (new record), Russia.

\section{ACKNOWLEDGMENTS}

We thank Prof. Yanko Kolarov of the Faculty of Pedagogie, University of Plovdiv, Bulgaria for providing useful comments. This work was supported by a grant from the National Institute of Biological Resources (NIBR), funded by the Ministry of Environment (MOE) of the Republic of Korea (NIBR No. 2013-02-001).

\section{REFERENCES}

Aubert JF, 1978. Les Ichneumonides ouest-palearctiques et leurs hotes 2. Banchinae et Suppl. aux Pimplinae. Laboratoire d'Evolution des Etres Organises, Paris \& EDIFAT-OPIDA, Echauffour, pp.1-318.

Capek M, 1955. [Einige Bemerkungen über die Parasitation der Raupen der Motte Tachyptilia disquei Meess.] Zoologicke Listy. Folia Zoologica, 4:29-32 (in Czech with Russian and German summaries).

Chao HF, 1980. [Description of Lycorina spilonotae Chao, sp. nov., with notes on its final-instar larvae (Hymenoptera: Ichneumonidae: Lycorinae)]. Entomotaxonomia, 2:165-168 (in Chinese with English summary).

Costa A, 1886a. Notizie ed osservazioni sulla geo-fauna Sarda. Memoria sesta. Risultamento delle ricerche fatte in Sardegna nella state del 1885. Atti della R. Accademia delle Scienze Fisiche et Matematiche, 2:1-40.

Costa A, 1886b. Notizie ed osservazioni sulla geo-fauna Sarda. Memoria sesta. Ricerche fatte ne' mesi di luglio ed agosto 1885. Rendiconto dell'Accademia della Scienze Fisiche et Matematiche, 25:51-53.

Cresson ET, 1874. Descriptions of Mexican Ichneumonidae. Proceedings of the Academy of Natural Sciences of Philadelphia, 1873:374-413.

Cushman RA, 1920. The North American Ichneumon-flies of the tribes Lycorini, Polysphinctini, and Theroniini. Proceedings of the United States National Museum, 58:7-48.

Cushman RA, Rohwer SA, 1920. Holarctic tribes of the Ichneumon-flies of the subfamily Ichneumoninae (Pimplinae). Proceedings of the United States National Museum, 57:379-396.

Doerksen GP, Neunzig HH, 1974. The biology of the appleleaf skeletonizer, Psorosina hammondi, on Crateaegus (Lepidoptera: Pyralidae: Phycitinae). Annals of the Entomological Society of America, 67:146-147.

Finlayson T, 1976. Cephalic structures and spiracles of finalinstar larvae of the genus Toxophoroides (Hymenoptera: Ichneumonidae: Lycorininae). Canadian Entomologist, 108: 981-984.

Friese G, 1963. Die Parasiten der paläarktischen Yponomeutidae (Lepidoptera, Hymenoptera, Diptera). Beiträge zur Entomologie, 13:311-326.

Fulmek L, 1968. Parasitinsekten der Insektengallen Europas. Beiträge zur Entomologie, 18:719-952.

He JH, Chen XX, Ma Y, 1996. Hymenoptera: Ichneumonidae. Economic insect fauna of China. Science Press, Beijing, pp. 1-697.

Holmgren AE, 1859. Conspectus generum Pimplariarum Sueciae. Öfversigt af Kongliga Vetenskaps-Akademiens Förhandlingar, 16:121-132.

Jonaitis VP, 1992. 7 new and 1 rare for Lithuania Ichneumonid species (Hymenoptera, Ichneumonidae) found in 1967-1987. In: New and rare for Lithuania insect species: records and descriptions of 1992 (Ed., Jonaitis VP). Institute of Ecology, Vilnius, pp. 1-115. 
Kasparyan DR, Khalaim AI, 2007. Pimplinae, Tryphoninae, Eucerotinae, Xoridinae, Agriotypinae, Lycorininae, Neorhacodinae, Ctenopelmatinae, Phrudinae, Ophioninae, Acaenitinae, Collyriinae, Mesochorinae. In: Key to the insects of russia Far Ease. Vol. IV. Neuropteroidea, Mecoptera, Hymenoptera (Ed., Lelej AS). Dalnauka, Vladivostock, pp. 11052 (in Russian).

Kleine R, 1909. Die Schmarotzer der Cerambyciden und Buprestiden. Entomologische Blätter, 5:177-179, 207-212.

Meyer NF, 1934. Schlupfwespen die in Russland in den letzten Jahren aus Schädlingen gezogen sind. Zeitschrift für Angewandte Entomologie, 20:611-618.

Postner M, 1954. Zur Biologie und Bekämpfung des kleinen Pappelbockes Saperda populnea L. (Cerambycidae). Zeitschrift für Angewandte Entomologie, 36:156-177.

Scheidter F, 1917. Über die Eiablage von Saperda populnea L. Naturwissenschaftliche Zeitschrift für Land- und Forstwirtschaft, 15:113-138.

Schiødte JC, 1839. Beretning om Resultaterne af en i Sommeren 1838 foretagen entomologisk Undersøgelse af det sydlige Sjaelland, en Deel af Laaland, og Bornholm. Naturhistorisk Tidsskrift, 2:309-394.

Sedivy J, 1986. The hosts of Ichneumon flies in Europe (Hymen- optera, Ichneumonidae). Acta Entomologica Bohemoslovaca, 83:10-23.

Seyrig A, 1932. Les Ichneumonides de Madagascar. I Ichneumonidae Pimplinae. Mémoires de l'Académie Malgache. Fascicule 11. L'Academie Malgache, Tananarive, pp. 1-183. Shaw MR, 2004. Notes on the biology of Lycorina triangulifera Holmgren (Hymenoptera: Ichneumonidae: Lycorininae). Journal of Hymenoptera Research, 13:302-308.

Uffeln K, 1940. In Westfalen gefangene und aus anderen Insekten erzogene Schlupfwespen. Abh. Landesmus. Naturkunde Province Westfalen Münster, 11:56-66.

Uhthoff-Kaufmann RR, 1991. The distribution and occurrence of the genus Saperda F. (Col.: Lamiidae) in Great Britain. Entomologist's Record, 103:129-134.

Yu DS, Van Achterberg C, Horstmann K, 2012. Taxapad 2012, Ichneumonoidea 2011. Database on flash-drive [Internet]. Dicky Sick Ki Yu, Ottawa, ON, Accessed 30 Oct 2013, <www.taxapad.com>. 